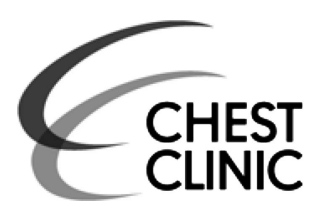
Cincinnati Children's Hospital Medical Center and the University of Cincinnati College of Medicine, Cincinnati, Ohio, USA

${ }^{2}$ Department of Pediatric Radiology, Texas Children's Hospital, Houston, Texas, USA

\section{Correspondence to}

Dr Alan S Brody, Department of Radiology, Cincinnati Children's Hospital Medical Center, Radiology MLC 5031, 3333 Burnet Avenue,

Cincinnati, $\mathrm{OH}$ 45229-3039, USA; alan.brody@cchmc.org

Accepted 4 April 2014 Published Online First 24 April 2014

\title{
Don't let radiation scare trump patient care: 10 ways you can harm your patients by fear of radiation-induced cancer from diagnostic imaging
}

\author{
Alan S Brody, ${ }^{1}$ R Paul Guillerman ${ }^{2}$
}

\section{RADIATION}

For many people, the term radiation is linked to atomic bombs, cancer and death. Even without these associations, ionising radiation in the form of $\mathrm{X}$-rays is frightening. Acute exposure to $\mathrm{X}$-rays cannot be sensed in any way, yet can be lethal within days or weeks if received at a high enough dose. There is clearly good reason to be concerned about unnecessary exposure to X-rays. In diagnostic imaging, balancing this danger is the benefit that can be realised from the use of these X-rays. When a group of leading general internists was asked to rank 30 medical innovations by the most adverse effect on their patients if the innovation were unavailable, the most important innovation by a considerable margin' was MRI and CT scanning. ${ }^{1}$ MRI does not use ionising radiation, but CT scanning is more widely available, can be completed in under $10 \mathrm{~s}$ compared with 20-30 min for MRI, and is the imaging modality of choice for acute trauma to the head or abdomen and for evaluation of the lungs.

How can your concern about radiation risk hurt your patient? Simply put, by not doing an imaging study that could benefit the patient more, or by unduly compromising image quality in an effort to reduce radiation dose. The following are 10 common ways that a medical caregiver can wrongly assess the risk : benefit of imaging to the detriment of his or her patients.

\section{Overweighting the risk of radiation}

The risk is very, very low. The lifetime attributable risk of cancer mortality to a 10 -year-old from a $3 \mathrm{mGy}$ average organ dose (similar to that from a body CT scan) is approximately $1 / 3000$ for a girl and1/4700 for a boy, using the Biological Effects of Ionising Radiation VII estimates. ${ }^{2}$ This compares to the $1 / 5$ probability, without additional radiation exposure, of dying of cancer for a typical person in the USA.

How can we understand or communicate this risk? A probability of $1 / 4000$ means that it will not occur $99.975 \%$ of the time. $1 / 4000$ is approximately the likelihood that a coin will come up heads 12 times in a row. It is about twice the annual probability of dying in an automobile collision in the USA, where there is about one fatality per 100 million vehicle miles travelled. ${ }^{3}$

Due to cognitive biases, unlikely outcomes tend to be overweighted and almost certain outcomes tend to be underweighted, ${ }^{4}$ so that the perceived likelihood of radiation-induced cancer is overweighted and the perceived likelihood of not getting a radiation-induced cancer is underweighted. Risks tend to be irrationally exaggerated when the risks are unknown or very low, delayed in effect, sensationalised, and not under control of the subject, all of which apply in the setting of radiation exposure from diagnostic imaging.

Applying population-based risk estimates to the radiation doses incurred to individual patients by diagnostic radiology exams

Statements from expert committees do not agree on the magnitude or precision of risk estimates, or even whether there is a risk at all in the diagnostic imaging range. The risks of medical imaging using a radiation dose less than $50 \mathrm{mSv}$ at one time (more than 15 times the dose from the above hypothetical CT scan) or $100 \mathrm{mSv}$ in multiple doses over a short period of time are too low to be detectable and may be non-existent, according to a 2011 position statement of the American Association of Physicists in Medicine (AAPM).

\section{Disregarding uncertainties and inconsistencies in published risk estimates}

Prospective estimates of cancer induced by medical radiation should include a statement that the estimates are highly speculative because of various uncertainties, according to a 2013 position statement of the International Organisation for Medical Physics. ${ }^{6}$ Taking into consideration the uncertainties in the estimated dose, the dose-response models, and the variability among patients, the error in estimating cancer risk to an individual from the radiation from a CT scan may be $500 \%$ or greater. ${ }^{7}$ Published articles provide even greater disparities in estimates of risk. The American Journal of Respiratory and Critical Care Medicine published two papers that estimated the risk from CT scanning in patients with cystic fibrosis. One estimated the likelihood of dying of cancer due to diagnostic CT imaging to be $13 \% .{ }^{8}$ The second estimated the risk of developing radiation-induced cancer at less than $0.5 \% .^{9}$ Cancer mortality is approximately $50 \%$, so this second paper suggests a risk of less than $0.25 \%$ of dying from CT radiation. Thus one paper suggests that the mortality risk is 50 times greater than the other. Both papers were written by well regarded researchers and included a medical physicist among the authors. 
If unrepaired, a DNA double-strand break (DSB) induced by radiation may be an initiating event for carcinogenesis. Large inter-individual variability in DNA DSB break repair has been observed in human fibroblasts before and after exposure to low doses of ionising radiation. ${ }^{10}$ This suggests that the DNA repair capacity of an individual rather than the radiation dose may be the primary determinant of risk at the dose range encountered in diagnostic radiology. ${ }^{11}$ Clinical disorders with impaired DNA DSB repair and elevated radiation-induced cancer risk include ataxia-telangiectasia and Nijmegen breakage syndrome.

\section{Comparing future risks with current benefits}

Comparing the risk of future cancer with the current benefit from an imaging study is not a like-for-like comparison. The risk from CT radiation is the risk of an event that, if it occurs, will occur many years in the future. A loss of life expectancy calculation provides a more meaningful estimate of risk than does mortality. Epidemiologic data indicate that radiation-induced cancers occur after a latency period, and at a similar age as naturally occurring cancers, ${ }^{12}$ predominantly at ages $45-85$. If the likelihood of dying of radiation-induced cancer is $1 / 4000$, the mean age of cancer development is 65 years (halfway between 45 and $85)$, the mean age of cancer death is 70 years, and the projected lifespan is 85 years, then the expected loss of life expectancy from a radiation-induced cancer is 15 years. The population-averaged loss of life expectancy is 15 years $\times 1$ / $4000=1 / 267$ of a year, or less than 2 days.

CT scans are often obtained in patients with life-threatening diseases, and if disease-related mortality is taken into consideration, the risk of radiation-induced cancer and the loss of life expectancy from radiation-induced cancer are smaller since these patients may not live long enough to develop these cancers. ${ }^{13}$ In a study of CT in young adults the observed risk of a patient dying within 5 years from their underlying disease was one to two orders of magnitude greater than the theoretical risk of dying from a cancer induced by $\mathrm{CT}^{14}$

\section{Accepting lower quality diagnostic information to reduce radiation dose}

In 2012, six paediatric radiology groups at sites with special interest and expertise in radiation dose reduction collaborated to develop diagnostic reference levels for abdominal CT scanning. Five percent of the CT scans using standard clinical protocols and radiation doses below the 25 th percentile were judged to be non-diagnostic, ${ }^{15}$ suggesting that 1 in 20 paediatric abdominal CT scans performed at some of the top institutions in the USA were inadequate for diagnostic purposes due to excessive radiation dose reduction efforts. This is wasted radiation exposure and, even worse, may negatively influence care when it results in misguided treatment based on inadequate or erroneous information.

Radiation dose minimisation and diagnostic optimisation are not synonymous, and can be contrary. For example, identifying early acute appendicitis on a CT scan after an equivocal clinical or ultrasound evaluation avoids ruptured appendicitis and the near-term consequences of peritonitis, sepsis, prolonged hospitalisation, and the longer-term consequences of future bowel obstruction due to adhesions. A decision analysis study using Markov modelling showed that ultrasound alone is the least cost-effective approach and ultrasound followed by CT when ultrasound is negative or non-diagnostic is the most costeffective approach for diagnosis of paediatric appendicitis, taking into consideration diagnostic performance, radiation-induced cancer risk, and appendicitis-related morbidity and mortality. ${ }^{16}$

\section{Failing to recognise flawed logic}

Recommendations on how to reduce risk are often poorly supported by data or based on flawed logic. Much concern has been raised about repetitive CT scanning in young patients. However, this usually occurs in very ill patients, such as those with cancer, bone marrow transplants, ventriculoperitoneal shunts, or cystic fibrosis, many of whom have a shortened life expectancy and are unfortunately likely to die before radiation-induced cancers would develop. There is reluctance among many radiologists to perform additional CT scans in these patients out of fear of excessive radiation exposure, but this represents a sunk cost bias. Previous radiation exposure is irrecoverable, and radiation-induced cancer risks related to prior imaging should not influence the risk-benefit analysis for subsequent imaging. ${ }^{17}$ Not performing a clinically indicated CT due to the perception of too much prior irradiation is irrational and more likely to cause harm than good. ${ }^{18}$

\section{Unreasonably frightening patients and families}

In a recent study, only $70 \%$ of parents were found to be willing or very willing to proceed with a head CT on their child thought to be necessary by the emergency department physician for evaluation of head injury after being informed of the risk of radiation-induced cancer, compared with $90 \%$ before being informed ${ }^{19}$ This risk aversion is particularly troubling given the well established benefit of CT in this setting. The yield (1-8\%) of detecting acute traumatic brain injury on a paediatric head CT following clinical decision rules at the point of care $^{20}$ is more than $100 \times$ greater than the theoretical future likelihood of radiation-induced cancer.

\section{Not realising that 'incidental' findings alone may confer a favourable risk : benefit balance}

The risk of radiation is so low compared with the yield of CT that the identification of highly important incidental findings may be more likely than the development of later cancer. In a recent study published in the journal Pediatrics, ${ }^{21}$ the incidence of incidental findings requiring immediate attention in children who had a CT scan for blunt head trauma was found to be $0.14 \%$, or approximately 1 in 700 . This likelihood of an urgent incidental finding is several times greater than the hypothetical probability of inducing a cancer from a paediatric head CT scan.

\section{Choosing the imaging modality based on radiation risk}

MRI scanning of the brain is commonly recommended as an alternative to CT because MRI does not use ionising radiation. Due to the longer imaging time, however, sedation is often required for MRI of infants and young children. The risks of sedation and anaesthesia are rarely considered in comparisons of CT and MRI. In a study of 30037 paediatric sedations, there were desaturations below $90 \%$ in $1.5 \%$, unexpected apnoea in $0.2 \%$ and laryngospasm in $0.04 \%$. The authors noted that the organisations that were studied had 'highly motivated and organised sedation services', ${ }^{22}$ suggesting that the risks at many institutions will be greater.

Perhaps more concerning is recent evidence that anaesthetic drugs may cause permanent damage to the infant brain. In a study that compared 383 children who underwent inguinal hernia repair before age 3 with 5050 children with no history of hernia repair, those who had hernia repair (and consequently exposure to anaesthetic agents) were 1.3-4.1 (95\% CIs) times 
more likely to have developmental delay or behaviour problems. An expert group report from the British Journal of Anaesthesia stated that 'a large body of preclinical and some retrospective clinical evidence suggest that exposure to general anaesthesia could be detrimental to cognitive development in young subjects'. ${ }^{23}$ The decision to choose between CT and MRI must consider far more than just the potential risk of ionising radiation from CT.

\section{Focusing resources on radiation reduction rather than more impactful priorities}

We all wish that we could address every issue that impacts the health of our patients. We cannot, and so we must prioritise those issues that have the greatest impact. A study published in the New England Journal of Medicine in 2010 found that medical errors 'causing or contributing to a patient's death' occurred in 6 of 1000 hospitalisations, and 64\% of these errors were preventable. More important, the study found that between 2002 and 2007 there had been no decrease in preventable harm. ${ }^{24}$ Clearly, preventable medical errors are far more likely to cause death than CT scanning, and these deaths are often acute rather than occurring many decades later.

\section{Competing interests None.}

Provenance and peer review Not commissioned; internally peer reviewed.

\section{REFERENCES}

1 Fuchs VR, Sox HC Jr. Physicians' views of the relative importance of thirty medical innovations. Health Aff 2001;20:30-42.

2 National Research Council (US). Committee to Assess Health Risks from Exposure to Low Level of lonizing Radiation. Health risks from exposure to low levels of ionizing radiation: BEIR VII Phase 2. Washington, DC: National Academies Press, 2006: xvi, 406p.

3 US Department of Transportation Administration NHTS. 2011 Data, DOT HS 811 753. 2013.

4 Kahneman D, Tversky A. Prospect theory — analysis of decision under risk. Econometrica 1979;47:263-91.

5 AAPM Position Statement on Radiation Risks from Medical Imaging Procedures. The American Association of Physicists in Medicine, 2011. http://www.aapm.org/org/ policies/details.asp?id=318\&type=PP\&current=true (accessed 14 Apr 2014).

6 IOMP Policy Statement No. 3. International Organization for Medical Physics. http:// www.iomp.org/sites/default/files/policy_statement_3.pdf (accessed 14 Apr 2014).
7 Martin CJ. Effective dose: how should it be applied to medical exposures? $\mathrm{Br} J$ Radiol 2007;80:639-47.

8 de Jong PA, Mayo JR, Golmohammadi K, et al. Estimation of cancer mortality associated with repetitive computed tomography scanning. Am J Respir Crit Care Med 2006:173:199-203.

9 de Gonzalez AB, Kim KP, Samet JM. Radiation-induced cancer risk from annual computed tomography for patients with cystic fibrosis. Am J Respir Crit Care Med 2007;176:970-3.

10 Wilson PF, Nham PB, Urbin SS, et al. Inter-individual variation in DNA double-strand break repair in human fibroblasts before and after exposure to low doses of ionizing radiation. Mutat Res-Fund Mol M 2010;683:91-7.

11 Mothersill C, Seymour CB. Radiation-induced bystander effects and the DNA paradigm: an 'out of field' perspective. Mutat Res 2006;597:5-10.

12 Preston $\mathrm{DL}$, Ron $\mathrm{E}$, Tokuoka $\mathrm{S}$, et al. Solid cancer incidence in atomic bomb survivors: 1958-1998. Radiat Res 2007;168:1-64.

13 Brenner DJ, Shuryak I, Einstein AJ. Impact of reduced patient life expectancy on potential cancer risks from radiologic imaging. Radiology 2011;261:193-8.

14 Zondervan RL, Hahn PF, Sadow CA, et al. Body CT scanning in young adults: examination indications, patient outcomes, and risk of radiation-induced cancer. Radiology 2013;267:460-9.

15 Goske MJ, Strauss KJ, Coombs LP, et al. Diagnostic reference ranges for pediatric abdominal CT. Radiology 2013;268:208-18.

16 Wan MJ, Krahn M, Ungar WJ, et al. Acute appendicitis in young children: cost-effectiveness of US versus CT in diagnosis-a Markov decision analytic model. Radiology 2009;250:378-86.

17 Eisenberg JD, Harvey HB, Moore DA, et al. Falling prey to the sunk cost bias: a potential harm of patient radiation dose histories. Radiology 2012; 263:626-8.

18 Eisenberg JD, Lewin SO, Pandharipande PV. The Fisherman's cards: how to address past and future radiation exposures in clinical decision making. AJR Am J Roentgenol 2014;202:362-7.

19 Boutis K, Cogollo W, Fischer J, et al. Parental knowledge of potential cancer risks from exposure to computed tomography. Pediatrics 2013;132:305-11.

20 Macias CG, Sahouria JJ. The appropriate use of CT: quality improvement and clinical decision-making in pediatric emergency medicine. Pediatr Radiol. 2011:41(Suppl 2):498-504.

21 Rogers AJ, Maher CO, Schunk JE, et al. Incidental findings in children with blunt head trauma evaluated with cranial CT scans. Pediatrics 2013; 132:e356-63.

22 Cravero JP, Blike GT, Beach $M$, et al. Incidence and nature of adverse events during pediatric sedation/anesthesia for procedures outside the operating room: report from the Pediatric Sedation Research Consortium. Pediatrics 2006;118:1087-96.

23 Jevtovic-Todorovic $\mathrm{V}$, Absalom AR, Blomgren $\mathrm{K}$, et al. Anaesthetic neurotoxicity and neuroplasticity: an expert group report and statement based on the BJA Salzburg Seminar. Br J Anaesth 2013;111:143-51.

24 Landrigan $\mathrm{CP}$, Parry GJ, Bones $\mathrm{CB}$, et al. Temporal trends in rates of patient harm resulting from medical care. N Engl J Med 2010;363:2124-34. 